Lenice Harumi Ishitani'

Glaura da Conceição Franco"

Ignez Helena O liva Perpétuo"'

Elisabeth Françaiv
Gerência de Epidemiologia e Informação. Secretaria Municipal de Saúde. Belo Horizonte, MG, Brasil

" Departamento de Estatística. Instituto de Ciências Exatas. Universidade Federal de Minas Gerais (UFMG). Belo Horizonte, MG, Brasil

II Departamento de Demografia. Faculdade de Ciências Econômicas. UFM G. Belo Horizonte, MG, Brasil

IV Departamento de Medicina Preventiva e Social. Faculdade de Medicina. UFM G. Belo Horizonte, M G, Brasil

Correspondência | Correspondence: Elisabeth França

Departamento de Medicina Preventiva e Social

Faculdade de Medicina - UFMG

Av. Alfredo Balena, $19010^{\circ}$ andar 30130-100 Belo Horizonte, MG, Brasil

E-mail: efranca@medicina.ufmg.br

Recebido: 2/6/2005 Revisado: 24/2/2006 Aprovado: 5/3/2006

\section{Desigualdade social e mortalidade precoce por doenças cardiovasculares no Brasil}

\author{
Socioeconomic inequalities and \\ premature mortality due to \\ cardiovascular diseases in Brazil
}

\section{RESU MO}

OBJETIVO: Investigar associação entre alguns indicadores de nível socioeconômico e mortalidade de adultos por doenças cardiovasculares no Brasil.

MÉTODOS: Foram analisados os óbitos de adultos (35 a 64 anos), ocorridos entre 1999 a 2001, por doenças cardiovasculares, e pelos subgrupos das doenças isquêmicas do coração e doenças cerebrovasculares-hipertensivas, obtidos no Sistema de Informação sobre Mortalidade. Foram selecionados para análise 98 municípios brasileiros, com melhor qualidade de informação. Para analisar a associação entre indicadores socioeconômicos e a mortalidade por doenças cardiovasculares, foi utilizada a regressão linear simples e múltipla.

RESULTADOS: Na análise univariada, verificou-se associação negativa para a mortalidade por doenças cardiovasculares e o subgrupo das cerebrovasculareshipertensivas com renda e escolaridade, e associação direta com taxa de pobreza e condições precárias de moradia. Quanto às doenças isquêmicas, houve associação inversa com taxa de pobreza e escolaridade, e direta com condições precárias de moradia. A escolaridade, após ajuste pelo modelo de regressão linear múltipla, permaneceu associada à mortalidade pela doença investigada e seus subgrupos. A cada ponto percentual de aumento na proporção de adultos com alta escolaridade, a taxa de mortalidade por doenças cardiovasculares diminui em 3,25 por 100.000 habitantes.

CONCLUSÕES: A análise da mortalidade dos municípios mostrou que a associação entre doenças cardiovasculares e fatores socioeconômicos é inversa, destacando-se a escolaridade. É provável que melhor escolaridade possibilite melhores condições de vida e, conseqüentemente, impacto positivo na mortalidade precoce.

DESCRITO RES: Doenças cardiovasculares, mortalidade. Fatores socioeconômicos. Fatores de risco. Desigualdades em saúde.

\section{ABSTRACT}

OBJECTIVE: To investigate the relationship between some indicators of socioeconomic status and adult mortality due to cardiovascular diseases in Brazil.

METHODS: Adult deaths (aged between 35 and 64 years) due to cardiovascular diseases and subgroups of ischemic heart disease and cerebrovascular-hypertensive 
diseases were assessed during the period from 1999 to 2001. Data was obtained from the National Mortality Information System. Ninety-eight Brazilian municipalities with the best quality of information were included in the study. Simple and multiple linear regression methods were used to assess the association between socioeconomic indicators and mortality due to cardiovascular diseases.

RESULTS: Univariate analysis revealed a negative association between mortality due to cardiovascular and cerebrovascular-hypertensive diseases and income and education and a direct association with poverty rates and poor housing conditions. In regard to ischemic heart disease, there was an inverse association with poverty rates and education indicators and a direct association with poor housing conditions. After adjusting to other variables, education still remained associated to mortality due to cardiovascular disease and its subgroups. For each percent point increase in adults with high level of education, there was a decrease of 3.25 per 100,000 inhabitants in the mortality rate due to cardiovascular diseases.

CONCLUSIONS: The analysis of mortality in those municipalities has shown there is an inverse association between cardiovascular diseases and social and economic factors, especially education. Is it likely that better education can improve life conditions and thus have a positive impact on premature mortality.

\section{KEYW O RD S: Cardiovascular diseases, mortality. Socioeconomic factors.} Risk factors. Health inequalities.

\section{INTRO DUÇÃO}

As doenças cardiovasculares (DCV) representam a primeira causa de morte no Brasil. Apesar da tendência de redução dos riscos de mortalidade por DCV no País e no mundo, algumas projeções indicam o aumento de sua importância relativa em países de baixa e média renda. A maior longevidade, associada ao possível aumento da incidência das DCV por adoção dos modos de vida com maior exposição a fatores de risco, são consideradas as principais razões deste incremento. Como fatores de risco estão o tabagismo e inatividade física, além de dieta rica em gorduras saturadas, com conseqüente aumento dos níveis de colesterol e hipertensão. ${ }^{10}$

No Brasil, um fato que agrava esse quadro é que, aproximadamente, um terço dos óbitos por DCV ocorrem precocemente em adultos na faixa etária de 35 a 64 anos.* Nesta faixa etária, as principais causas de óbito por doenças do aparelho circulatório são as doenças isquêmicas do coração, as doenças cerebrovasculares e as doenças hipertensivas. Ressalte-se que essas causas são em grande parte evitáveis, diante da probabilidade de diminuição da ocorrência dessas mortes, se houver assistência ou prevenção oportunas. ${ }^{15}$

Apesar da importância crescente das DCV nos países em desenvolvimento, são poucos os estudos que investigam como as desigualdades sociais afetam o quadro de mortalidade. Em países desenvolvidos, existem evidências de uma relação inversa entre nível socioeconômico e a incidência e/ou prevalência e mortalidade por DCV. ${ }^{7}$ Estudos ecológicos realizados em diferentes países ${ }^{19,21}$ e investigações brasileiras que abordaram diferenciais intra-urbanos ${ }^{4,18}$ verificaram maior taxa de mortalidade por DCV em populações de pior nível socioeconômico. Entretanto, em outras investigações brasileiras ${ }^{5,13}$, ** verificouse maior risco de morte por DCV em macrorregiões socioeconomicamente desenvolvidas, corroborando Cooper $^{3}$ que coloca ser este o padrão encontrado em países ainda não desenvolvidos.

Uma possível explicação para esses diferentes resultados está relacionada ao problema da qualidade da informação. Enquanto os estudos realizados em municípios analisaram dados de mortalidade de melhor qualidade, o mesmo não ocorreu com os estudos, de maior abrangência, das unidades federativas e das macrorregiões, muito heterogêneas do ponto de vista socioeconômico. As regiões Norte e Nordeste do Brasil são consideradas menos desenvolvidas e com maiores deficiências na oferta, no acesso e na organização dos serviços de saúde. Assim, suas informações sobre mortalidade são comprometidas pela captação defi- 
ciente de óbitos e pelas elevadas proporções de causas mal definidas informadas no Sistema de Informação sobre Mortalidade (SIM). O Sul e o Sudeste, ao contrário, possuem melhor qualidade das estatísticas vitais e apresentam maiores taxas de mortalidade, possivelmente devido a melhor cobertura do SIM. A comparação dos dados entre as regiões é prejudicada pelo problema do sub-registro e da qualidade da informação relativa às causas de óbito, situação geralmente associada à pobreza.*

Questiona-se, dessa forma, se uma análise de municípios brasileiros com melhor qualidade da informação também não encontraria resultados semelhantes aos de países desenvolvidos. Considerando a precocidade dos óbitos ocorridos em adultos, que representam importantes perdas de anos potenciais de vida produtivos, o estudo da mortalidade por DCV assume aspecto fundamental. Dessa forma, o presente estudo teve por objetivo analisar a associação entre mortalidade de adultos por doenças cardiovasculares e indicadores socioeconômicos em municípios brasileiros com melhor qualidade de informação.

\section{MÉTO DOS}

Trata-se de estudo ecológico, cuja unidade de análise foram municípios selecionados de todas as macrorregiões do Brasil. As variáveis dependentes ou resposta foram as taxas de mortalidade por doenças do aparelho circulatório ou cardiovasculares (DCV), e pelos subgrupos das doenças isquêmicas do coração (DIC) e doenças cerebrovasculares e hipertensivas $(\mathrm{DCDH})$. As variáveis independentes ou preditoras foram os indicadores socioeconômicos.

Os critérios para seleção dos municípios consideraram o problema da instabilidade das taxas e da qualidade da informação, que diz respeito ao conhecido sub-registro de óbitos e às deficiências na certificação das causas de morte no Brasil. ${ }^{13}$ Para minimizar o problema de instabilidade das taxas, foram inicialmente selecionados 224 municípios que possuíam população maior que 100.000 habitantes. Desses, foram identificados 180 que apresentavam pelo menos $90 \%$ da população residindo em área urbana, onde se supõe que a questão do sub-registro de óbitos seja menor.

Procurou-se assegurar melhor qualidade do registro das causas de óbito na declaração de óbito (DO), admitindo-se uma proporção máxima de $10 \%$ de causas mal definidas, reduzindo-se para 113 municípios. Outro critério de seleção utilizado foi adaptado do estudo de Szwarcwald et al, ${ }^{20}$ que considera como indicador de regularidade da informação os dados que apresentaram variação menor que $10 \%$ dos coeficientes de mortalidade geral (padronizados por idade) em relação à média, em um período de três anos. Esse processo resultou na seleção de 98 municípios, com maior representação das regiões Sudeste e Sul com 49 e 25 municípios, respectivamente. Foram ainda selecionados 12 municípios da região Nordeste, oito da Centro-Oeste e quatro da região Norte.

Os dados populacionais do Censo Demográfico 2000 e as estimativas de populações para 1999 e 2001 foram obtidos do Datasus/MS.** Em $90 \%$ deles, houve predomínio da população adulta feminina (35 a 64 anos), que variou de $45,6 \%$ a $56,3 \%$ (mediana de $52,0 \%$ ).

Foram analisados os óbitos por doenças cardiovasculares ocorridos no período de 1999 a 2001, em adultos de 35 a 64 anos, disponibilizados pelo SIM. Esse sistema de abrangência nacional foi implantado em 1975 pelo Ministério da Saúde, com o objetivo de obter dados padronizados para produção de indicadores de saúde.

As causas de óbitos foram agrupadas conforme a Lista de Tabulação de Mortalidade $\mathrm{n}^{\circ} 1$, da Classificação Estatística Internacional de Doenças e Problemas Relacionados à Saúde - $10^{\mathrm{a}}$ Revisão (CID-10). ${ }^{16}$ Foram selecionadas para este estudo as doenças do aparelho circulatório ou cardiovasculares, relativas ao capítulo IX da CID-10 (códigos: I00-I99), destacando-se dois sub-grupos: 1) doenças isquêmicas do coração (códigos: I20-I25); 2) doenças hipertensivas e cerebrovasculares (códigos: I10-I13 e I60-I69). Esses dois subgrupos representam cerca de $75 \%$ dos óbitos por doenças cardiovasculares na faixa etária estudada e são consideradas causas evitáveis de morte. ${ }^{15}$ Optou-se pela análise conjunta das doenças hipertensivas e doenças cerebrovasculares, por esta última ser complicação da primeira, sendo freqüentemente mencionadas de forma associada na DO, conforme verificado em estudo recente de causas múltiplas de morte. ${ }^{17}$ Além disso, essas causas vêm sendo apresentadas de forma associadas nas listas de causas evitáveis de mortalidade. ${ }^{15}$

Para o cálculo das taxas de mortalidade por doenças cardiovasculares do período, considerou-se como numerador o número de óbitos do período e como denominador, o resultado da soma da população do Censo 2000 e estimativas para 1999 e 2001 . As taxas foram ajustadas por idade pelo método direto, consi- 
Tabela 1 - Indicadores socioeconômicos e taxas de mortalidade* por doenças cardiovasculares de municípios brasileiros selecionados. Brasil, 1999-2001.

\begin{tabular}{|c|c|c|c|c|c|c|c|c|c|}
\hline Variável & Média & DP & Mín & Máx. & P10 & P25 & P50 & P75 & P90 \\
\hline $\begin{array}{l}\text { Alta escolaridade }(\%) \\
\text { Renda per capita }(\mathrm{R} \$) \\
\text { Taxa pobreza }(\%) \\
\text { Índice Gini } \\
\text { Condições precárias moradia }(\%)\end{array}$ & $\begin{array}{l}11,4 \\
369,03 \\
19,3 \\
0,56 \\
20,6\end{array}$ & $\begin{array}{c}5,8 \\
134,12 \\
8,9 \\
0,06 \\
9,2\end{array}$ & $\begin{array}{c}1,1 \\
149,59 \\
2,9 \\
0,45 \\
5,2\end{array}$ & $\begin{array}{c}32,0 \\
834,00 \\
46,2 \\
0,69 \\
42,6\end{array}$ & $\begin{array}{c}4,1 \\
214,75 \\
9,1 \\
0,49 \\
11,1\end{array}$ & $\begin{array}{c}7,9 \\
281,65 \\
12,8 \\
0,53 \\
13,9\end{array}$ & $\begin{array}{c}10,7 \\
346,11 \\
16,9 \\
0,56 \\
18,9\end{array}$ & $\begin{array}{l}15,4 \\
431,58 \\
23,6 \\
0,61 \\
24,9\end{array}$ & $\begin{array}{c}18,6 \\
549,96 \\
31,5 \\
0,64 \\
37,6\end{array}$ \\
\hline $\begin{array}{l}\text { Variáveis dependentes } \\
\text { DCV (TM por } 100.000 \text { hab.) } \\
\text { DIC (TM por } 100.000 \text { hab.) } \\
\text { DCDH (TM por } 100.000 \text { hab.) }\end{array}$ & $\begin{array}{c}232,96 \\
84,17 \\
89,42\end{array}$ & $\begin{array}{l}48,31 \\
29,94 \\
22,97\end{array}$ & $\begin{array}{c}154,23 \\
22,38 \\
46,57\end{array}$ & $\begin{array}{l}375,32 \\
158,91 \\
162,69\end{array}$ & $\begin{array}{c}172,15 \\
48,73 \\
60,69\end{array}$ & $\begin{array}{c}197,33 \\
59,18 \\
71,87\end{array}$ & $\begin{array}{c}224,88 \\
83,46 \\
87,46\end{array}$ & $\begin{array}{l}269,97 \\
103,60 \\
103,01\end{array}$ & $\begin{array}{l}301,15 \\
126,92 \\
121,71\end{array}$ \\
\hline
\end{tabular}

Fonte: Censo Demográfico, 2000/IBGE

SIM/D atasus/M S

*Taxas por 100.000 habitantes

Alta escolaridade: Percentual de adultos de 25 anos ou mais com 12 ou mais anos de estudo, 2000

Condições precárias moradia: Percentual de pessoas que vivem em domicílios com densidade acima de duas pessoas por dormitório, 2000

P: Percentis

DCV: Doenças cardiovasculares

DIC: Doenças isquêmicas do coração

DCDH: Doenças cerebrovasculares e hipertensivas

derando como a população recenseada do Brasil em 2000 como padrão.

Os indicadores socioeconômicos de cada município selecionado para o estudo foram obtidos do Programa das Nações Unidas para o Desenvolvimento (PNUD), * calculados a partir do Censo Demográfico, 2000. Foram consideradas como indicadores socioeconômicos as seguintes variáveis:

a) Renda per capita: os valores, expressos em reais de $1^{\circ}$ de agosto de 2000, correspondem à razão entre o somatório da renda per capita de todos os indivíduos e o número total desses indivíduos. A renda per capita de cada indivíduo é calculada pela razão entre a soma da renda de todos os membros da família e o número de membros dessa família.

b) Taxa de pobreza: proporção de indivíduos com renda domiciliar per capita inferior a meio salário-mínimo (linha de pobreza), vigente em agosto de 2000.

c) Índice de Gini: medida do grau de desigualdade na distribuição da renda domiciliar per capita, varia de zero a um. Quando não há desigualdade de renda de todos os indivíduos seu valor é zero. Quando a desigualdade é máxima, ou seja, apenas um indivíduo detém toda a renda da sociedade e a renda de todos os outros indivíduos é nula, o valor deste indicador é um.

d) Alta escolaridade: percentual de pessoas de 25 anos ou mais que completaram pelo menos um ano de curso universitário em 2000 (12 anos ou mais de estudo).

e) Condições precárias de moradia: percentual de pessoas que vivem em domicílios com densidade acima de duas pessoas por dormitório.

Foi realizada análise de regressão linear simples e múl- tipla ${ }^{1}$ para avaliar a associação entre os indicadores socioeconômicos selecionados e a mortalidade por DCV, DIC e DCDH. Inicialmente, foram incluídas no modelo multivariado todas as variáveis que apresentaram significância estatística menor que 0,20 na análise univariada, permanecendo no modelo final aquelas que tiveram significância menor que 0,10. A adequação dos modelos foi testada pela análise de resíduos e estatísticas de ajuste, como o coeficiente de determinação ajustado $\left(\mathrm{R}^{2}\right)$. Foi utilizado o programa Stata 8.0 para processamento e análise estatística.

\section{RESU LTADOS}

Os indicadores socioeconômicos mostraram uma grande variação (Tabela 1). Quanto à escolaridade, observou-se que, em geral, é baixa. Em metade dos municípios, menos de $10,7 \%$ dos adultos de 25 anos e mais têm mais de 12 anos de estudo. Houve um município em que o nível superior foi alcançado por apenas $1,1 \%$ da população, sendo que esta proporção atingiu $32,0 \%$ em outra localidade.

Mais da metade dos municípios tiveram renda per capita menor que $\mathrm{R} \$ 346,11$ em 2000 , correspondendo a 2,3 salários-mínimos, e pelo menos $16,9 \%$ da população de metade dos municípios ainda vivem em condições abaixo da linha de pobreza. $O$ índice de Gini variou de 0,45 a 0,69, com média de 0,56, revelando a desigualdade de renda existente nos municípios.

Em relação às condições de moradia, observou-se que em metade dos municípios, cerca de $19 \%$ da população viviam em habitações com maior aglomeração de moradores e, em $10 \%$, quase $40 \%$ da população viviam nessas condições. 
Tabela 2 - Correlação entre as variáveis socioeconômicas e mortalidade por doenças cardiovasculares de municípios brasileiros selecionados. Brasil, 1999-2001.

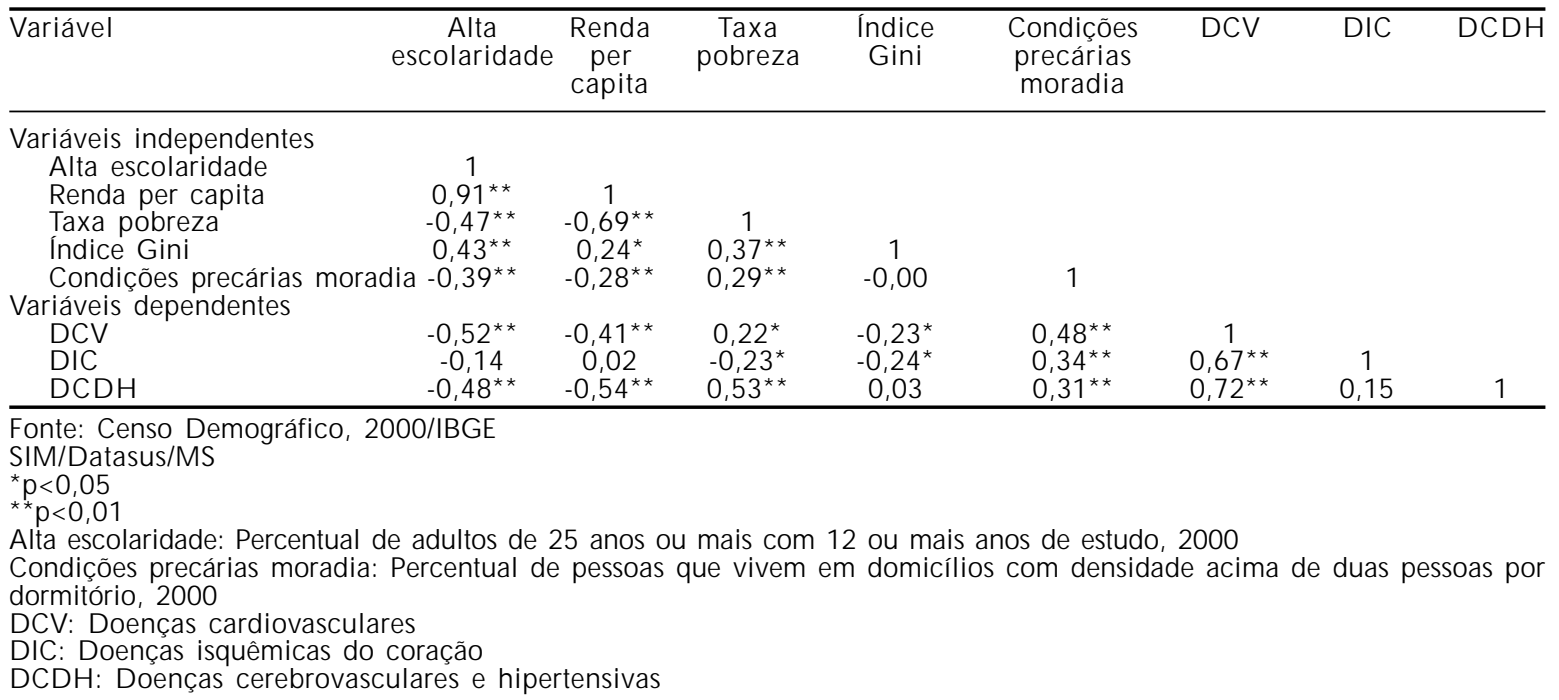

Os riscos de mortalidade por doenças cardiovasculares também apresentaram uma variação. Em relação às DIC, a maior taxa de mortalidade foi de 158,91 por 100.000 habitantes, sete vezes maior do que a do município com a menor taxa $(22,38$ por 100.000 habitantes). Para as $\mathrm{DCDH}$, a razão das taxas foi de 3,5 .

Verificou-se que, em geral, os indicadores socioeconômicos estão correlacionados significativamente entre si, principalmente a alta escolaridade e a renda per capita $(r=0,91)$. Quanto à correlação entre as variáveis independentes e dependentes, verificou-se uma correlação inversa com situação socioeconômica da população. Ou seja: a mortalidade por DCV e por $\mathrm{DCDH}$ foi maior quando eram piores a escolaridade e a renda (correlação inversa), e maiores a taxa de pobreza e a proporção de pessoas vivendo em condições precárias de moradia (correlação direta). $\mathrm{Na}$ mortalidade por DIC, houve relação inversa com a taxa de pobreza e correlação direta com as condições precárias de moradia. O índice de Gini mostrou correlação negativa com as DCV e DIC (Tabela 2).

A análise de regressão simples mostrou que pior condição socioeconômica está associada a maiores riscos de mortalidade por DCV e DCDH. Quanto às DIC, os resultados foram diferentes: não houve associação com renda per capita e a associação foi inversa com a taxa de pobreza (Tabela 3).

Na análise de regressão múltipla, a análise de resíduos mostrou um bom ajuste dos modelos para DCV e DIC. Apesar de a distribuição dos resíduos não ser normal no modelo da DCDH mesmo após transformação logarítmica, decidiu-se manter o modelo devido ao número de municípios analisados.
Verificou-se que a escolaridade (associação negativa) e piores condições de moradia (associação positiva) permaneceram significativas para as DCV, o que não ocorreu com as outras variáveis. No modelo final, 34\% da variação total de DCV pôde ser explicada pelo modelo de regressão $\left(\mathrm{R}^{2}\right.$ ajustado $\left.=0,34\right)$. Por este modelo, a cada ponto percentual de aumento na proporção de adultos com alta escolaridade, diminui em 3,25 por 100.000 habitantes a taxa de mortalidade por DCV (Tabela 3 ).

Já em relação às DIC, as variáveis socioeconômicas significativas que permaneceram no modelo final $\left(\mathrm{R}^{2}\right.$ ajustado $=0,24$ ) foram as relativas à maior escolaridade (associação negativa), taxa de pobreza (associação negativa) e piores condições de moradia (associação positiva) (Tabela 3 ).

Quanto às DCDH, o modelo mais bem ajustado $\left(\mathrm{R}^{2}\right.$ ajustado $=0,34$ ) foi a combinação da escolaridade (associação negativa) e taxa de pobreza (associação positiva) (Tabela 3 ).

\section{DISCUSSÃO}

No presente estudo, observou-se associação inversa entre condições socioeconômicas (indicadas pela renda, escolaridade e moradia) e mortalidade por DCV e DCDH. A associação com taxa de pobreza foi direta, conforme esperado. Quanto às DIC, também houve associação inversa com maior escolaridade e condições de moradia. Entretanto, essa associação observada entre DIC e taxa de pobreza não era esperada. Os valores baixos de $\mathrm{R}^{2}$ observados nos três modelos sugerem, no entanto, que outras variáveis além das socioeconômicas provavelmente estão associadas à mortalidade por doenças cardiovasculares. 
Estudos ecológicos como é o caso da presente investigação, são relevantes do ponto de vista da saúde pública, já que o contexto social exerce importante influência na definição dos modos de viver. É possível que a análise por modelos hierárquicos permitisse maior conhecimento da associação investigada, uma vez que os indicadores socioeconômicos dos municípios são influenciados pelo contexto social do nível mais agregado, as diversas macrorregiões do País.

Da mesma forma que no presente trabalho, vários outros estudos ecológicos ${ }^{4,18,19,21}$ também evidenciaram associação negativa entre mortalidade e situação socioeconômica. Nos Estados Unidos ${ }^{19}$ e na Austrália, ${ }^{21}$ assim como nos municípios do Rio de Janeiro $^{18}$ e de São Paulo, ${ }^{4}$ populações residentes em áreas de melhor nível socioeconômico tiveram menor mortalidade por DCV, DIC e DCDH. Ainda no Brasil, em estudo realizado em oito capitais, ${ }^{*}$ verificou-se correlação negativa entre mortalidade por doenças cerebrovasculares e situação socioeconômica, principalmente em mulheres. A maior mortalidade por doenças cardiovasculares em situação socioeconô- mica menos privilegiada também foi comprovada em estudos realizados em nível individual. ${ }^{2,9}$

No Brasil, alguns estudos que analisaram dados relativos ao nível de macrorregiões e unidades federadas verificaram maior mortalidade por DCV em regiões socioeconomicamente mais desenvolvidas, ${ }^{5,13, * *}$ sendo observadas taxas de mortalidade por DCV significativamente maiores nos Estados com menores taxas de pobreza. ${ }^{5}$ Estes resultados não são concordantes com os da presente investigação, provavelmente devido à utilização de unidades de análise de diferentes níveis que dificultam a comparação. É possível que o problema do sub-registro diferencial de óbitos e a qualidade da informação sobre causas de morte interfiram nos resultados das análises de mortalidade em estudos de abrangência nacional. No presente trabalho, a utilização de critérios que assegurassem seleção de municípios com melhor qualidade da informação resultou em achados compatíveis com os de vários estudos. ${ }^{4,7,19,21}$ Isso pode indicar que ocorrem desigualdades na mortalidade por doenças cardiovasculares relacionadas a fatores socioeconômicos no País.

Tabela 3 - Associação entre indicadores socioeconômicos e taxas de mortalidade por doenças cardiovasculares, isquêmicas do coração e cerebrovasculares e hipertensivas em 98 municípios brasileiros selecionados, 1999-2001.

\begin{tabular}{|c|c|c|c|c|c|}
\hline Variável & Coeficiente & erro-padrão & $p>|t|$ & $\mathrm{F}$ & $\mathrm{R}^{2}$ adj \\
\hline \multicolumn{6}{|l|}{$\begin{array}{l}\text { D oenças cardiovasculares } \\
\text { Regressão simples }\end{array}$} \\
\hline \multicolumn{6}{|l|}{ Regressão simples } \\
\hline Renda per capita & $-0,15$ & 0,03 & 0,00 & & 0,16 \\
\hline Taxa de pobreza & 1,20 & 0,54 & 0,03 & & 0,04 \\
\hline Índice de Gini & $-1,96$ & 0,83 & 0,02 & & 0,05 \\
\hline Condições precárias moradia & 2,52 & 0,47 & 0,00 & \multirow{4}{*}{0,00} & 0,22 \\
\hline Regressão múltipla & & & & & 0,34 \\
\hline Alta escolaridade & $-3,25$ & 0,75 & 0,00 & & \\
\hline Condições precárias moradia & 1,71 & 0,47 & 0,00 & & \\
\hline \multicolumn{6}{|l|}{ Doenças isquêmicas do coração } \\
\hline Alta escolaridade & $-0,71$ & 0,52 & 0,18 & \multirow{9}{*}{0,00} & \multirow{9}{*}{$\begin{array}{c}0,01 \\
-0,01 \\
0,05 \\
0,05 \\
0,10 \\
0,24\end{array}$} \\
\hline Renda per capita & 0,00 & 0,02 & 0,88 & & \\
\hline Taxa de pobreza & $-0,79$ & 0,33 & 0,02 & & \\
\hline Índice de Gini & $-1,25$ & 0,51 & 0,02 & & \\
\hline Condições precárias moradia & 1,10 & 0,31 & 0,00 & & \\
\hline Regressão múltipla & & & & & \\
\hline Alta escolaridade & $-1,01$ & 0,55 & 0,07 & & \\
\hline Taxa de pobreza & $-1,47$ & 0,34 & 0,00 & & \\
\hline Condições precárias moradia & 1,26 & 0,32 & 0,00 & & \\
\hline \multicolumn{6}{|c|}{ Doenças cererebrovasculares e hipertensivas } \\
\hline \multicolumn{6}{|l|}{ Regressão simples } \\
\hline Alta escolaridade & $-1,92$ & 0,35 & 0,00 & \multirow{8}{*}{0,00} & \multirow{8}{*}{$\begin{array}{c}0,23 \\
0,28 \\
0,27 \\
-0,01 \\
0,09 \\
0,34\end{array}$} \\
\hline Renda per capita & $-0,09$ & 0,01 & 0,00 & & \\
\hline Taxa de pobreza & 1,37 & 0,22 & 0,00 & & \\
\hline Índice de Gini & 0,10 & 0,40 & 0,80 & & \\
\hline Condições precárias moradia & 0,79 & 0,24 & 0,00 & & \\
\hline \multicolumn{4}{|l|}{ Regressão múltipla } & & \\
\hline Alta escolaridade & $-1,19$ & 0,37 & 0,00 & & \\
\hline Taxa de pobreza & 1,00 & 0,24 & 0,00 & & \\
\hline
\end{tabular}

Fonte: Censo Demográfico, 2000/IBGE; SIM/Datasus/MS

Alta escolaridade: Percentual de adultos de 25 anos ou mais com 12 ou mais anos de estudo, 2000

Condicỗes precárias moradia: Percentual de pessoas que vivem em domicílios com densidade acima de duas pessoas por dormitório, 2000

*Lotufo PA. A mortalidade precoce por doenças crônicas nas capitais de regiões metropolitanas do Brasil [tese de doutorado]. São Paulo: Faculdade de Saúde Pública da Universidade de São Paulo; 1996.

**Ministério da Saúde, Secretaria de Vigilância em Saúde, Departamento de Análise de Situação de Saúde. Saúde Brasil 2004 - uma análise da situação de saúde. Brasília (DF); 2004. 
Indicadores socioeconômicos relativos à renda, apesar de apresentarem limitações, são importantes marcadores da situação socioeconômica. Melhor renda proporciona acesso a bens e serviços. Já a desigualdade de renda, uma medida relativa, aborda o problema da distribuição de riqueza e está diretamente associada com a mortalidade. Uma das explicações é que a carência relativa pode gerar frustração que leva à deterioração da saúde: o indivíduo de baixa renda se sentirá pior numa sociedade onde há maior desigualdade. ${ }^{12} \mathrm{~A}$ escolaridade, relacionada a modos de viver que promovem a saúde, é um indicador com algumas vantagens em relação à renda: há um baixo percentual de não-resposta e na idade adulta, dificilmente sofre alterações. As condições de moradia refletem a situação socioeconômica de uma população, sendo consideradas preditoras da mortalidade. ${ }^{7}$

Dentre as variáveis socioeconômicas analisadas, a escolaridade foi um importante indicador associado à mortalidade por DCV. A variável "renda per capita", fortemente correlacionada à escolaridade, perdeu a significância estatística na análise multivariada, o que ocorreu também no caso das DCDH. Maior renda oferece maior oportunidade de acesso a bens e serviços, inclusive educação de qualidade e assistência de saúde com recursos diagnósticos e terapêuticos eficazes, incluindo profissionais qualificados e equipamentos sofisticados de diagnóstico. ${ }^{7} \mathrm{~A}$ associação direta verificada das DCV e DCDH, no presente estudo, com a taxa de pobreza pode estar relacionada ao acesso aos serviços de saúde, devido à associação negativa entre o número de médicos por habitante e a taxa de pobreza. ${ }^{5}$

Fatores que aumentam o risco de DCV, como a hipertensão arterial, a hipercolesterolemia, o tabagismo e a inatividade física, são mais freqüentes em grupos de menor escolaridade, conforme observado em estudos de base individual. ${ }^{9}$ A presença simultânea de dois ou mais desses fatores também tem sido observada em pessoas com menor escolaridade. ${ }^{11}$ No Brasil, Freitas et $\mathrm{al}^{6}$ verificaram que a prevalência de hipertensão arterial foi mais alta em tabagistas, em quem não praticava regularmente atividade física e em analfabetos. Esse quadro é agravado pelo fato desses indivíduos possuírem menor conhecimento sobre prevenção de doenças crônicas, apontando a necessidade de ações preventivas direcionadas para a população mais carente. Entretanto, nossa realidade é ainda bem diversa. A procura dos serviços de saúde por motivos preventivos é maior para a população de melhor poder aquisitivo, enquanto que para os indivíduos mais pobres, é maior por motivo de doenças. ${ }^{14}$

A correlação positiva entre o índice de Gini com a alta escolaridade e a associação inversa com a mortalidade por DCV e DIC foi diferente do observado em países desenvolvidos. ${ }^{8}$ Como é provável que os municípios brasileiros selecionados apresentem melhor nível socioeconômico que os não selecionados, tal achado sugere que o índice de Gini tem comportamento diferente do de países desenvolvidos. Uma hipótese é que, como maior escolaridade é um indicador de melhor situação socioeconômica, esta variável paradoxalmente passa a se comportar como um indicador de desigualdade, por ser somente acessível a uma minoria da população (mediana de $10,7 \%$ para os municípios). Outro achado não correspondente com o de países desenvolvidos foi a associação negativa da taxa de pobreza com as DIC, enfatizando a necessidade de outros estudos que investiguem melhor a relação entre situação socioeconômica e mortalidade por DIC. Esse resultado pode estar relacionado ao problema da qualidade da informação sobre renda: a resistência da população em fornecer dados sobre rendimentos reduz a confiabilidade dessa informação. ${ }^{7}$ Outra possível explicação é o maior acesso da população socioeconomicamente mais privilegiada aos recursos diagnósticos, resultando em melhor registro das causas de óbito.

Concluindo, os resultados encontrados indicam que a mortalidade por doenças cardiovasculares acomete principalmente populações menos privilegiadas socioeconomicamente. É provável que maior escolaridade também possibilite melhor captação das mensagens de promoção da saúde, com melhor resposta às campanhas educativas. Neste aspecto, deve ser dispensada atenção especial ao público com menor escolaridade com elaboração de mensagens adequadas. Dessa forma, evita-se que as estratégias de prevenção alarguem ainda mais as diferenças de mortalidade entre os níveis socioeconômicos, beneficiando aqueles de melhor nível educacional e de renda, que irão adotar "modos de vida" saudáveis.

\section{AGRADECIMENTOS}

À Professora Dra. Sandhi Maria Barreto, da Faculdade de Medicina da UFMG, pelas sugestões. 


\section{REFERÊN CIAS}

1. Altman DG. Practical statistics for medical research. Londres: Chapman \& Hall; 1991.

2. Borrell C, Regidor E, Arias LC, Navarro P, Puigpinós $\mathrm{R}$, Domínguez $\mathrm{V}$, et al. Inequalities in mortality according to educational level in two large Southern European cities. Int J Epidemiol. 1999;28:58-63.

3. Cooper RS. Social inequality, ethnicity and cardiovascular disease. Int J Epidemiol. 2001;30(Suppl 1):S48-52.

4. Drumond Júnior M, Barros MBA. Desigualdades socioespaciais na mortalidade do adulto no município de São Paulo. Rev Bras Epidemiol. 1999;2(1-2):34-49.

5. Duarte EC, Schneider MC, Paes-Sousa R, Ramalho WM, Sardinha LMV, Silva Júnior JB, et al. Epidemiologia das desigualdades em saúde no Brasil: um estudo exploratório. Brasília (DF): OPAS; 2002.

6. Freitas OC, Carvalho FR, Neves JM, Veludo PK, Parreira RS, Gonçalves RM, et al. Prevalence of hypertension in the urban population of Catanduva, in the State of São Paulo, Brazil. Arq Bras Cardiol. 2001;77(1):16-21.

7. Kaplan GA, Keil JE. Socioeconomic factors and cardiovascular disease: a review of the literature. Circulation. 1993;88(4 Pt 1):1973-98.

8. Kennedy BP, Kawachi I, Prothrow-Stith D. Income distribution and mortality: cross sectional ecological study of the Robin Hood index un the United States. BMJ. 1996;312:1004-7.

9. Kilander L, Berglund L, Boberg M, Vessby B, Lithell $H$. Education, lifestyle factors and mortality from cardiovascular disease and cancer: a 25-year followup of Swedish 50-year-old men. Int J Epidemiol. 2001;30:1119-26.

10. Lenfant C. Can we prevent cardiovascular diseases in low and middle-income countries? Bull World Health Organ. 2001;79:980-2.
11. Lessa I, Araújo MJ, Magalhães L, Almeida Filho N, Aquino E, Costa MCR. Simultaneidade de fatores de risco cardiovascular modificáveis na população adulta de Salvador (BA), Brasil. Rev Panam Salud Pública. 2004;16(2):131-7.

12. Macinko JA, Shi L, Starfield B, Wulu Jr JT. Income inequality and health: a critical review of the literature. Med Care Res Rev. 2003;60:407-52

13. Mello Jorge MHP, Gotlieb SLD, Laurenti R. A saúde no Brasil: análise do período 1996 a 1999. Brasília (DF): O PAS/OMS; 2001.

14. Neri M, Soares W. Desigualdade social e saúde no Brasil. Cad Saúde Pública. 2002;18(Supl):77-87.

15. Nolte $E$, McKee M. Does healthcare save lives? Avoidable mortality revisited. London: Nuffield Trust; 2004.

16. O rganização Mundial de Saúde. Classificação estatística internacional de doenças e problemas relacionados à saúde. 10ar rev. São Paulo: Edusp; 1993.

17. Rezende EM, Sampaio IBM, Ishitani LH. Causas múltiplas de morte por doenças crônicodegenerativas: uma análise multidimensional. Cad Saúde Pública. 2004;20:1223-31.

18. Santos SM, Noronha CP. Padrões espaciais de mortalidade e diferenciais socioeconômicos na cidade do Rio de Janeiro. Cad Saúde Pública. 2001;17:1099-110.

19. Singh GK, Siahpush M. Increasing inequalities in allcause and cardiovascular mortality among US adults aged 25-64 years by area socioeconomic status, 1969-1998. Int / Epidemiol. 2002;31:600-13.

20. Szwarcwald CL, Leal MC, Andrade CLT, Souza Junior PRB. Estimação da mortalidade infantil no Brasil: 0 que dizem as informações sobre óbitos e nascimentos do Ministério da Saúde? Cad Saúde Pública. 2002;18:1725-36.

21. Turrell G, Mathers $C$. Socioeconomic inequalities in all-cause and specific-cause mortality in Australia: 1985-1987 and 1995-1997. Int J Epidemiol. 2001;30:231-9. 